See discussions, stats, and author profiles for this publication at: https://www.researchgate.net/publication/267759545

\title{
Induced fractional valley number in graphene with topological defects
}

Article in Physical Review B · November 2014

DOI: 10.1103/PhysRevB.91.035404 . Source: arXiv

2 authors, including:

Marcelo Hott

São Paulo State University

57 PUBLICATIONS 581 CITATIONS

SEE PROFILE

Some of the authors of this publication are also working on these related projects:

Nonlinear Dirac equations View project

Vortices in Abelian Maxwell-Chern-Simons-Higgs model with two distinct gauge potentials View project 


\title{
Induced fractional valley number in graphene with topological defects
}

\author{
Angel E. Obispd* and Marcelo Hott \\ UNESP Universidade Estadual Paulista - Campus de Guaratinguetá - DFQ. \\ 12516-410, Guaratinguetá-SP, Brazil.
}

\begin{abstract}
We report on the possibility of valley number fractionalization in graphene with a topological defect that is accounted for in Dirac equation by a pseudomagnetic field. The valley number fractionalization is attributable to an imbalance on the number of one particle states in one of the two Dirac points with respect to the other and it is related to the flux of the pseudomagnetic field. We also discuss the analog effect the topological defect might lead in the induced spin polarization of the charge carriers in graphene.

PACS numbers: 71.10.Pm,73.22.Pr,81.05.ue,61.48.Gh,11.10.Kk
\end{abstract}

*Electronic address: ovasquez@feg.unesp.br

${ }^{\dagger}$ Electronic address: marcelo.hott@pq.cnpq.br 


\section{INTRODUCTION}

Approximately seven years ago it was demonstrated by Hou, Chamon and Mudry [1] that fermion charge fractionalization can take place in monolayer graphene due to Kekulé distortions that are described by means of a complex valued scalar field (Higgs field) coupled to the Dirac field which, in its turn, describes the dynamics of massless charge carriers in the quantum field theory obtained as the low-energy regime of a discrete model (tightbinding Hamiltonian) of nearest-neighbor hopping on the two-dimensional honeycomb lattice [2, 3]. The mechanism for charge fractionalization relies upon the existence of zero-energy eigenstates bounded to the vortex associated to the Kekule distortions that also would open an energy gap at the (two) previously present degeneracy points - two inequivalent Dirac points in the Brillouin zone where the valence and the conduction bands intersect each other - of the tight-binding Hamiltonian. While the so-called valley symmetry is preserved by such midgap bound-states, the vorticity of the distortion, either $n \geq 1$ or $n \leq-1$, determines which one of the two triangular sublattices supports $|n|$ zero modes. That is, the zero modes do not exhibit the also called pseudospin (sublattice) symmetry presented in both the tightbinding and Dirac Hamiltonians. The zero modes and their sole contribution to the fermion charge fractionalization were shown to persist in a chiral gauge theory for graphene proposed by Jackiw and Pi [4] by adding an axial-vector gauge potential that incorporates axial gauge symmetry $\left(U_{A}(1)\right)$ to the field theoretical model in [1].

The chiral coupling of electrons with gauge field in [4] was motivated to give a dynamical origin of the Higgs field, which together with the gauge potential, enters in a phenomenological model (Landau-Ginzburg-Abrikosov-Nielsen-Olesen model), whose minimum energy solutions exhibit vortex-like profiles, as those proposed to be realized in graphene. It worths to mention that a chiral gauge field was introduced much earlier in the Dirac equation, as an effective equation for charge carriers in a single layer of Carbon atoms to describe frustration in fullerenes [5]. Later, Pachos, Stone and Temme [6] shown that in a honeycomb lattice the modulated hopping strength, otherwise described by means of a real scalar field, may become a modulated complex-valued field if one considers that the hexagonal lattice has its topology altered, such that the former planar system becomes spherical, as in fullerenes. To take into account the modified topology at the theoretical level, one introduces a chiral gauge field in the Dirac Hamiltonian that describes the low-energy dynamics of charge carriers. 
In this way, [6] has provided a physical realization of magnetic vortices with fractionalized charge, as those proposed originally in [1] and [4] and also analyzed in detail in [7] and in [8] by taking into account the effects of external magnetic fields.

In a remarkable work [3] dedicated to the role gauge fields might play in the dynamics of charge carriers described by an effective quantum field theory of Dirac fermions, Semenoff showed that parity anomaly, a phenomenon that was shown to happen in Quantum Electrodynamics in $2+1$ space-time dimensions [9], could be simulated in a planar honeycomb lattice with two species of atoms. In that scenario there is a difference in energies of electrons localized on the different atoms and that implies in a parity violating "mass term" to the field theory effective Hamiltonian that is eventually responsible for the anomalous current that, paraphrasing Semenoff, "would couple to an unphysical external field of abnormal parity and is therefore not directly observable". In other words, that was an axial-vector gauge potential entering in scene, at least theoretically, in condensed matter, specially in graphite monolayer. Nowadays, it has been proposed that very intense pseudomagnetic fields can be induced by strain in graphene nanobubbles [10] and such pseudomagnetic fields might be associated to the curl of an axial-vector gauge potential non-minimally coupled to Dirac spinors describing the dynamics of electrons in strained graphene.

Indeed, fictitious magnetic fields in graphene have been very fruitful both theoretically and experimentally, as for example, in the modeling of corrugations and elastic deformations and in the study of influences that topological defects could lead in the electronic properties of graphene [11]-[17]; in the experimental realization of Landau levels in very intense (up to $300 T$ ) pseudomagnetic fields due to stress in strained graphene [10] and the possibility to observe, also by means of a scanning-tunneling-microscopy, Aharonov-Bohm interferences due to local deformations (microstresses) in graphene [18]. A good review on gauge fields in graphene can be found in [19], where the introduction at theoretical level of elastic deformations, topological defects and curvature in the low-energy effective Hamiltonian for graphene is reviewed and some of their effects in the electronic properties are discussed (see also [20] and [21]). Additionally, fictitious magnetic fields have been proposed as possible valleys filters in strained graphene [22]- [23]; a valley filter allows the transmission of a current associated to only one of the valleys, while filters the other one, that is, it transmits a valley-polarized current and constitutes a crucial mechanism in valleytronics [24], as much as spin-filtering mechanism is fundamental to spintronics. 
Motivated by many of the works cited above, we have shown [25] that vector and axialvector gauge potentials by themselves can bind zero-energy electrons and that fractional charge may be induced even in the absence of Kekule distortions. In this vein, we have also discussed the relation of such induced fractional charge to the parity anomaly which would be realized in gapped graphene as proposed almost thirty years ago [3] and in gapped graphene whose parity symmetry breaking term is provided by the Haldane energy [26]. In addition we also discussed the possible fractionization of another induced quantum number, which we had called chiral charge (number) in the presence of magnetic and pseudomagnetic fields and had briefly shown the connection of the chiral charge to the time-component of the abnormal current found in [3].

The chiral charge (number) seems to have an important physical meaning and must be treated as a physical observable in graphene whenever the valley symmetry is preserved at the quantum Hamiltonian level. It is more appropriate to call it valley number, instead. Since the valley symmetry is manifest in this system and there is no intervalley scattering, there is a doubling of fermions for each energy value and it is legitimate to assign to each one-particle state a label (index) to account for the fermion doubling. By its turn, the valley number is defined here as the net number of valley states, i.e. $N_{\mathrm{v}}=N_{+}-N_{-}$, where $N_{+}$is the total number of states (summed over all energy states) around to one of the Dirac points, while $N_{-}$is the total number of states associated to the other Dirac point.

Here, we consider the sample of graphene in the presence of an external uniform magnetic field and with a local topological defect of disclination type, which is equivalent to a pseudomagnetic field typical of a very thin and long solenoid, the very same magnetic field usually used to discuss the Aharonov-Bohm (AB) effect [27]-[28]. We show that the induced valley number is due only to the zero-energy modes (zero modes) of one-particle states and that there is an imbalance in the number of zero-energy eigenstates associated to the two Dirac points; as a consequence, the induced net valley number is shown to be given by $N_{v}= \pm 1 / 2(1-2\{\Phi / 2 \pi\})$, where $0<\{\Phi / 2 \pi\}<1$ is the fractional part of the reduced pseudomagnetic flux, whereas the global sign \pm arises from the freedom to choose the zero-energy particles either in the conduction $(+)$ or in the valence $(-)$ band. Some interesting features of this result are, the independence of the valley number on the applied external magnetic field, the fractionalization (even irrational values) of the valley number for $\{\Phi / 2 \pi\} \neq 1 / 2$, and its null result for $\{\Phi / 2 \pi\}=1 / 2$. Moreover, by extending the concept 
of valley-filtering further, we could say that this is a case of valley-polarized vacuum due to a partial valley-filtering, when some of the zero-energy localized states of one of the valleys are filtered.

In the next section we discuss the one-particle states by solving the proper Dirac equation we have in hands and comment on the relevant symmetries of the problem. In the third section we present the second quantization of the fermion field, comment on the manifest valley-symmetry in the context of quantum field theory, calculate the induced valley number and show that one may have quasiparticles carrying fractional valley charge. We also compute the induced electric charge in this context.

To show the similarity of that supposed partial valley-filtering to a partial spin-filtering also in graphene, in the fourth section we consider the very same configurations of magnetic and pseudomagnetic fields in the effective Hamiltonian for low-energy electrons whenever the valley degree of freedom is decoupled and the relevant degrees of freedom are the pseudospin (associated to the two triangular sublattices) and the spin polarization. There we do not take into account the antiferromagnetic order as it was considered in [29], because that would break the spin symmetry at the Hamiltonian level, what would not give reliability to the spin polarization of the field as a physical observable. We show that the filtering of some zero-energy states of spin polarization implies into quasiparticles carrying fractional, even irrational, spin polarization. The fifth section is left to further comments on the results we have found and a short analysis about the possible induction of fractional valley number in strained and in in-plane deformed graphene samples.

\section{BOUND STATES ON MAGNETIC AND PSEUDOMAGNETIC FIELDS}

The effective field theory Hamiltonian describing the dynamics of the electrons on the graphene honeycomb structure in the presence of an external magnetic field $\overrightarrow{B_{V}}=\vec{\nabla} \times \vec{V}$, and a pseudomagnetic field $\vec{B}_{A}=\vec{\nabla} \times \vec{A}$ can be written as

$$
\mathcal{H}=\int d^{2} \vec{r} \Psi^{\dagger}(\vec{r}, t) \hat{h}_{D} \Psi(\vec{r}, t)
$$

where $\hat{h}_{D}=\vec{\alpha} \cdot\left(-i \vec{\nabla}-e \vec{V}-\gamma_{5} \vec{A}\right)$ is the Dirac Hamiltonian operator and $\Psi(\vec{r})$ is a fourcomponent spinor, whose transpose is $\Psi^{T}=\left(\psi_{+}^{b} \psi_{+}^{a} \psi_{-}^{a} \psi_{-}^{b}\right)$. The superscripts $a$ and $b$ in the spinor components designate the triangular sublattices where the electrons are supported on, 
while the subscripts \pm stand for each one of the two inequivalent Dirac points. The matrix structure in (11) is made explicit by means of the following matrices

$$
\begin{aligned}
\beta & =\left(\begin{array}{ll}
0 & I \\
I & 0
\end{array}\right), \vec{\alpha}=\left(\begin{array}{cc}
\vec{\sigma} & 0 \\
0 & -\vec{\sigma}
\end{array}\right), \\
\alpha^{3} & =\left(\begin{array}{cc}
\sigma_{3} & 0 \\
0 & -\sigma_{3}
\end{array}\right), \gamma_{5}=-i \alpha^{1} \alpha^{2} \alpha^{3}=\left(\begin{array}{cc}
I & 0 \\
0 & -I
\end{array}\right),
\end{aligned}
$$

where $I$ is the $2 \times 2$ identity matrix and $\vec{\sigma}=\left(\sigma_{1}, \sigma_{2}\right)$ and $\sigma_{3}$ are the Pauli matrices in the standard representation.

We notice that (11) is invariant under local $U(1) \times U_{A}(1)$ gauge transformations $\Psi \rightarrow$ $e^{i\left(e \vartheta(\vec{x})+\gamma_{5} \omega(\vec{x})\right)} \Psi, \quad \vec{V} \rightarrow \vec{V}+\vec{\nabla} \vartheta(\vec{x})$ and $\vec{A} \rightarrow \vec{A}+\vec{\nabla} \omega(\vec{x})$. Moreover, because we are in $(2+1)$ dimensions, the chiral anomaly is absent.

The corresponding time-independent Dirac equation

$$
\hat{h}_{D} \Psi(\vec{r}, t)=E \Psi(\vec{r}, t)
$$

can be decomposed in two independent Dirac equations, for the upper $\psi_{+}^{T}=\left(\psi_{+}^{b} \psi_{+}^{a}\right)$ and lower $\psi_{-}^{T}=\left(\psi_{-}^{a} \psi_{-}^{b}\right)$ components of the energy eigenfunctions, that are associated to each one of the Dirac points in the Brillouin zone of the honeycomb lattice:

$$
\left[( \pm \vec{\sigma}) \cdot \vec{\Pi}_{ \pm}\right] \psi_{ \pm}(\vec{r}, t)=E \psi_{ \pm}(\vec{r}, t)
$$

with $\vec{\Pi}_{ \pm}=-(i \vec{\nabla}+e \vec{V} \pm \vec{A})$.

The bispinors $\Psi_{+}^{T}=\left(\psi_{+}^{T} 0\right)$ and $\Psi_{-}^{T}=\left(0 \psi_{-}^{T}\right)$ are also eigenstates of $\gamma_{5}$ that commutes with the Dirac Hamiltonian operator, with eigenvalues \pm 1 , respectively. This is the manifestation of the valley symmetry at the quantum mechanics level. Furthermore, once the Dirac Hamiltonian operator anticommutes with $\alpha^{3}$ and with $\alpha^{3} \gamma_{5}$, the energy spectrum is symmetric around the zero-energy level. In other words, if there is a norm-preserving operator (unitary operator $\mathcal{C}$ ) such that $\left\{\hat{h}_{D}, \mathcal{C}\right\}=0$, then for each positive-energy normalized eigenstate, $\Psi_{|E|}$, there is one corresponding negative-energy normalized eigenstate $\Psi_{-|E|}=\mathcal{C} \Psi_{|E|}$ and the zero modes are self-conjugate.

When the external magnetic field is uniform and one considers that the graphene sheet is under disclination, the vector and axial-vector potentials can be written in the symmetric 
gauges, respectively as:

$$
V^{i}=-\frac{B}{2} \varepsilon^{i j} x^{j}, A^{i}=-\frac{\Phi}{2 \pi r^{2}} \varepsilon^{i j} x^{j}
$$

where $\varepsilon^{12}=-\varepsilon^{21}=1$ and $\Phi=\int d^{2} \vec{r} B_{A}$ is the flux of the pseudomagnetic field $B_{A}=$ $\Phi \delta(r) / 2 \pi r$ (in cylindrical coordinates), which exhibits the same profile of the magnetic field used to describe the AB effect. Such effect involves a charged particle in the presence of a background magnetic field concentrated within a flux tube where the probability of the particle be found is zero. Here it is the topological defect that is represented by such (pseudo)magnetic field (see [27]-[28])

The zero-energy normalized eigenfunctions associated to the electrons under the above magnetic and pseudomagnetic fields are given by

$$
\begin{aligned}
& \Psi_{0, l,+}(\vec{r})=\sqrt{\frac{|e B / 2|^{1+l-\frac{\Phi}{2 \pi}}}{\pi \Gamma\left(1+l-\frac{\Phi}{2 \pi}\right)}}\left(\begin{array}{c}
e^{i l \theta} \\
0 \\
0 \\
0
\end{array}\right) r^{l-\frac{\Phi}{2 \pi}} e^{-\frac{|e B|}{4} r^{2}} \text { and } \\
& \Psi_{0, k,-}(\vec{r})=\sqrt{\frac{|e B / 2|^{1+k+\frac{\Phi}{2 \pi}}}{\pi \Gamma\left(1+k+\frac{\Phi}{2 \pi}\right)}}\left(\begin{array}{c}
0 \\
0 \\
e^{i k \theta} \\
0
\end{array}\right) r^{k+\frac{\Phi}{2 \pi}} e^{-\frac{|e B|}{4} r^{2}},
\end{aligned}
$$

for $e B>0$, and by

$$
\begin{aligned}
& \Psi_{0, l,+}(\vec{r})=\sqrt{\frac{|e B / 2|^{1+l-\frac{\Phi}{2 \pi}}}{\pi \Gamma\left(1+l-\frac{\Phi}{2 \pi}\right)}}\left(\begin{array}{c}
0 \\
e^{-i l \theta} \\
0 \\
0
\end{array}\right) r^{l-\frac{\Phi}{2 \pi}} e^{-\frac{|e B|}{4} r^{2}} \text { and } \\
& \Psi_{0, k,-}(\vec{r})=\sqrt{\frac{|e B / 2|^{1+k+\frac{\Phi}{2 \pi}}}{\pi \Gamma\left(1+k+\frac{\Phi}{2 \pi}\right)}}\left(\begin{array}{c}
0 \\
0 \\
0 \\
e^{-i k \theta}
\end{array}\right) r^{k+\frac{\Phi}{2 \pi}} e^{-\frac{|e B|}{4} r^{2}}
\end{aligned}
$$

for $e B<0$. In the above expressions, $\theta$ is the angular variable in cylindrical coordinates and $l, k \in \mathbb{Z}$, with $l>\Phi / 2 \pi-1$ and $k>-\Phi / 2 \pi-1$, which are conditions to get normalized 
states. From those conditions one can note that there are infinite zero modes in both valleys, and that the pseudomagnetic field causes the lower bound of $k$ to be less than that of $l$ in the case $\Phi>0$. This lead us to conjecture that there are additional states of $\Psi_{0, k,-}$ with respect to $\Psi_{0, l,+}$, and vice-versa in the case $\Phi<0$.

Solutions of the Dirac equations as those in (4) for the scattering of massive fermions (when a mass term $m \sigma^{3}$ is added to the Dirac Hamiltonian operator) by only an ABlike magnetic field were discussed in several papers, for instance [30]-[32] and of massless fermions in [33]. In our case, the presence of the uniform magnetic field change the energy spectrum of the particle from a continuous spectrum to a discrete one. Our treatment to reach the excited states, $E \neq 0$, follows the one given in [34, 35], where it is discussed the wavefunctions of massive fermions in the presence of an uniaxial magnetic field with two contributions a uniform magnetic field and the AB-like magnetic field. Parenthetically we notice that each one of the equations in (4) corresponds exactly to the equation solved in [34, 35], except that we are interested in massless fermions and that the ground states were obtained here through the first-order differential equations (4), while the second-order differential equations are used in [34, 35] to obtain all the eigenstates, but the irregular ones at the origin. (For the sake of simplicity we use a specific value of the adjoint extension parameter; any other choice does not modify the main results presented in the next section).

The orthonormal eigenstates of positive energy, angular momentum and of $\gamma_{5}$ can be worked out straightforwardly and have the forms

$$
\begin{aligned}
& \Psi_{\left|E_{+}\right|, l,+}(\vec{r})=\frac{e^{i l \theta}}{\sqrt{2}}\left(\begin{array}{c}
R_{n, \nu_{+}}(r) \\
i R_{n-1, \nu_{+}+1}(r) \\
0 \\
0
\end{array}\right), \\
& \Psi_{\left|E_{-}\right|, k,+}(\vec{r})=\frac{e^{i k \theta}}{\sqrt{2}}\left(\begin{array}{c}
0 \\
0 \\
R_{n, \nu_{-}}(r) \\
-i R_{n-1, \nu_{-}+1}(r)
\end{array}\right),
\end{aligned}
$$

where $\nu_{+}=l-\Phi / 2 \pi, \nu_{-}=k+\Phi / 2 \pi$, and

$$
R_{n, \nu}(r)=\sqrt{\frac{(|e B| / 2)^{1+\nu} n !}{\pi \Gamma(n+\nu+1)}} r^{\nu} e^{-\frac{|e B|}{4} r^{2}} L_{n}^{\nu}\left(\gamma r^{2}\right)
$$


are the Gauss Laguerre modes in cylindrical coordinates conveniently constructed such that $\int\left|R_{n, \nu}(r)\right|^{2} d^{2} \vec{r}=1$, and we are assuming that $R_{-1, \nu}(r)=0$ and $\nu>1$.

The bound states solutions for $\nu_{ \pm}<-1$ are given by

$$
\begin{aligned}
& \Psi_{\left|E_{+}\right|, l,+}(\vec{r})=\frac{e^{i l \theta}}{\sqrt{2}}\left(\begin{array}{c}
R_{n,\left|\nu_{+}\right|}(r) \\
-i R_{n,\left|\nu_{+}\right|-1}(r) \\
0 \\
0
\end{array}\right), \\
& \Psi_{\left|E_{-}\right|, k,-}(\vec{r})=\frac{e^{i k \theta}}{\sqrt{2}}\left(\begin{array}{c}
0 \\
0 \\
R_{n,\left|\nu_{-}\right|}(r) \\
i R_{n,\left|\nu_{-}\right|-1}(r)
\end{array}\right),
\end{aligned}
$$

while the eigenstates of negative energy with $\nu_{ \pm}>+1$ and $\nu_{ \pm}<-1$ can be obtained by applying the unitary operator $\alpha^{3}$ to the eigenstates (8) and (10), respectively.

The subscripts $\left|E_{ \pm}\right|$designate the energy eigenvalues which are the Landau levels (LL)

$$
\left|E_{ \pm}\right|=\sqrt{2 e B n}, \text { with } n=1,2,3, \ldots \text { for }\left\{\begin{array}{l}
l \geqslant 2+[\Phi / 2 \pi] \\
k \geqslant 1-[\Phi / 2 \pi]
\end{array}\right.
$$

that are obtained from the regularity condition $\nu_{ \pm}>+1$ while, from $\nu_{ \pm}<-1$ one obtains

$$
\left|E_{ \pm}\right|=\sqrt{2 e B(n-m \pm \Phi / 2 \pi)}(n=0,1,2, \ldots,)
$$

where $m$ stands for $l \leqslant-1+[\Phi / 2 \pi]$ in case of $\left|E_{+}\right|$and for $k \leqslant-2-[\Phi / 2 \pi]$ in case of $\left|E_{-}\right|$, with $[\Phi / 2 \pi]>0$ representing the integer part of the reduced pseudomagnetic flux.

Only regular eigenfunctions have been considered so far. The irregular ones, corresponding to $\nu_{+}=-\{\Phi / 2 \pi\}, 1-\{\Phi / 2 \pi\}$ and to $\nu_{-}=\{\Phi / 2 \pi\},-1+\{\Phi / 2 \pi\},(0<\{\Phi / 2 \pi\}<1$ is the fractional part of the reduced pseudomagnetic flux) are obtained from the firstorder differential equations (44). We have found that the irregular eigenstates $\Psi_{\left|E_{+}\right|, 1+\left[\frac{\Phi}{2 \pi}\right],+}$ and $\Psi_{\left|E_{-}\right|,-\left[\frac{\Phi}{2 \pi}\right],-}$ have their corresponding energy eigenvalues in the set (11), whereas for $\Psi_{\left|E_{+}\right|,\left[\frac{\Phi}{2 \pi}\right],+}$ and $\Psi_{\left|E_{-}\right|,-1-\left[\frac{\Phi}{2 \pi}\right],-}$ the corresponding energy eigenvalues belong to the set (12).

Notice that the LL (including the zero-energy level) are degenerate with respect to eigenstates of $\gamma_{5}$ and $\hat{L}=-i \partial_{\theta}$. The zero-energy level, one has $l \geq[\Phi / 2 \pi]$ and $k \geq-1-[\Phi / 2 \pi]$, and the LL one has $l \geqslant 1+[\Phi / 2 \pi]$ and $k \geqslant-[\Phi / 2 \pi]$, due to the irregular eigenstates. The 
energy levels in the set (12) with $l \leqslant[\Phi / 2 \pi]$ and $k \leqslant-1-[\Phi / 2 \pi]$ may be degenerate only for $\{\Phi / 2 \pi\}=1 / 2$. Moreover, one only has a complete set of eigenstates if the zero-energy eigenstates with $l=[\Phi / 2 \pi]$ and $k=-1-[\Phi / 2 \pi]$ are dropped out from the set of eigenstates. This complete set of eigenstates comprises the basis in which the fermion field operator will be built from in the next section.

\section{INDUCED VALLEY NUMBER}

In this section we focus in the computation of the induced valley number that is defined here as the vacuum expectation value of the valley number operator, namely

$$
\hat{N}_{\mathrm{v}}=\frac{1}{2} \int d^{2} \vec{r}\left[\hat{\Psi}^{\dagger}(\vec{r}, t), \gamma_{5} \hat{\Psi}(\vec{r}, t)\right]
$$

where $\hat{\Psi}^{\dagger}(\vec{r}, t)$ is the fermion field operator that is expanded in the basis of the one-particle states presented in the previous section in the following way

$$
\begin{aligned}
\widehat{\Psi}(\vec{r}, t)= & \sum_{l=[\Phi / 2 \pi]+1}^{+\infty} \tilde{\mathbf{c}}_{0, l,+} \Psi_{0, l,+}(\vec{r})+\sum_{k=-[\Phi / 2 \pi]}^{+\infty} \tilde{\mathbf{c}}_{0, k,-} \Psi_{0, k,-}(\vec{r})+ \\
& +\sum_{n=0}^{+\infty}\left(\sum_{l}\left(\mathbf{c}_{n, l,+} \Psi_{\left|E_{+}\right|, l,+}(\vec{r}) e^{-i\left|E_{+}\right| t}+\mathbf{d}_{n, l,+}^{\dagger} \alpha^{3} \Psi_{\left|E_{+}\right|, l,+}(\vec{r}) e^{+i\left|E_{+}\right| t}\right)+\right. \\
& \left.+\sum_{k}\left(\mathbf{c}_{n, k,-} \Psi_{\left|E_{-}\right|, k,-}(\vec{r}) e^{-i\left|E_{-}\right| t}+\mathbf{d}_{n, k,-}^{\dagger} \alpha^{3} \Psi_{\left|E_{-}\right|, k,-}(\vec{r}) e^{+i\left|E_{-}\right| t}\right)\right) .
\end{aligned}
$$

In this expansion of the fermion field $\mathbf{c}_{n, m, \mathrm{v}}\left(\mathbf{d}_{n, m, \mathrm{v}}^{\dagger}\right)$ is the absorption (creation) operator of a particle of energy $\left|E_{ \pm}\right|$, angular momentum $m=l, k$ and valley $\mathrm{v}= \pm$ in the conduction (valence) band. The energies $\left|E_{ \pm}\right|$of the particles are given by expressions (11) and (12) with the corresponding values $l$ and $k$ can assume. The particles of zero-energy are, by construction of the fermion field, supposed to be in the conduction band and their absorption operators are represented by $\tilde{\mathbf{c}}_{0, m, \mathrm{v}}$. The creation $\mathbf{d}_{n, m, \mathrm{v}}^{\dagger}\left(\mathbf{c}_{n, m, \mathrm{v}}^{\dagger}\right)$ and absorption $\mathbf{d}_{n, m, \mathbf{v}}\left(\mathbf{c}_{n, m, \mathrm{v}}\right)$ operators of particles in valence (conduction) band obey the following anticommutation relations

$$
\begin{aligned}
& \left\{\mathbf{c}_{n, m, \mathrm{v}}, \mathbf{c}_{n^{\prime}, m^{\prime}, \mathrm{v}^{\prime}}^{\dagger}\right\}=\left\{\mathbf{d}_{n, i}, \mathbf{d}_{n^{\prime}, i^{\prime}}^{\dagger}\right\}=\delta_{n n^{\prime}} \delta_{m m^{\prime}} \delta_{\mathrm{vv}^{\prime}}, \\
& \left\{\tilde{\mathbf{c}}_{0, m, \mathrm{v}}, \tilde{\mathbf{c}}_{0, m^{\prime}, \mathrm{v}^{\prime}}^{\dagger}\right\}=\delta_{m m^{\prime}} \delta_{\mathrm{vv}^{\prime}},
\end{aligned}
$$


with all the others possible anticommutators vanishing. Then, one can show that $\left\{\widehat{\Psi}^{\dagger}(\vec{r}, t), \widehat{\Psi}\left(\vec{r}^{\prime}, t\right)\right\}=\delta^{2}\left(\vec{r}-\vec{r}^{\prime}\right)$, since one has a complete set of eigenfunctions as that developed in the previous section.

Then, the valley number operator can be expressed as $\hat{N}_{\mathrm{v}}=\hat{N}_{+}-\hat{N}_{-}$, where

$$
\hat{N}_{+}=\sum_{l=[\Phi / 2 \pi]+1}^{+\infty}\left(\tilde{\mathbf{c}}_{0, l,+}^{\dagger} \tilde{\mathbf{c}}_{0, l,+}-\frac{1}{2} \int d^{2} \vec{r}\left|\Psi_{0, l,+}\right|^{2}\right)+\sum_{n} \sum_{l=-\infty}^{+\infty}\left(\mathbf{c}_{n, l,+}^{\dagger} \mathbf{c}_{n, l,+}-\mathbf{d}_{n, l,+}^{\dagger} \mathbf{d}_{n, l,+}\right),
$$

and

$$
\hat{N}_{-}=\sum_{k=-[\Phi / 2 \pi]}^{+\infty}\left(\tilde{\mathbf{c}}_{0, k,-}^{\dagger} \tilde{\mathbf{c}}_{0, k,-}-\frac{1}{2} \int d^{2} \vec{r}\left|\Psi_{0, k,-}\right|^{2}\right)+\sum_{n} \sum_{k=-\infty}^{+\infty}\left(\mathbf{c}_{n, k,-}^{\dagger} \mathbf{c}_{n, k,-}-\mathbf{d}_{n, k,-}^{\dagger} \mathbf{d}_{n, k,-}\right)
$$

are the fermion number operator associated to the valley + and valley - , respectively. In obtaining these expressions one must remember that for each positive-energy state of a given valley there is a corresponding negative-energy state, such that they cancel each other. Furthermore, one can note that each of the fermion number operators acquires a $c$-number contribution whose origin is due to the zero modes, i.e.

$$
\begin{aligned}
c-\text { number }_{+} & =-\frac{1}{2} \int d^{2} \vec{r} \sum_{l=[\Phi / 2 \pi]+1}^{+\infty}\left|\Psi_{0, l,+}\right|^{2}=-\frac{1}{2} \int_{0}^{+\infty} d \zeta e^{-\zeta} \sum_{l=[\Phi / 2 \pi]+1}^{+\infty} \frac{\zeta^{l-\Phi / 2 \pi}}{\Gamma(1+l-q \Phi / 2 \pi)}= \\
& =-\frac{1}{2} \int_{0}^{+\infty} d \zeta\left(1+\frac{\zeta^{-\{\Phi / 2 \pi\}} e^{-\zeta}}{\Gamma(1-\{\Phi / 2 \pi\})}-\frac{\Gamma(1-\{\Phi / 2 \pi\}, \zeta)}{\Gamma(1-\{\Phi / 2 \pi\})}\right)= \\
& =-\frac{1}{2}\left(\{\Phi / 2 \pi\}+\int_{0}^{+\infty} d \zeta\right), \\
c-\text { number }_{-} & =-\frac{1}{2} \int d^{2} \vec{r} \sum_{k=-[\Phi / 2 \pi]}^{+\infty}\left|\Psi_{0, k,-}\right|^{2}=-\frac{1}{2} \int_{0}^{+\infty} d \zeta e^{-\zeta} \sum_{k=-[\Phi / 2 \pi]}^{+\infty} \frac{\zeta^{k+\Phi / 2 \pi}}{\Gamma(1+k+\Phi / 2 \pi)}= \\
& =-\frac{1}{2} \int_{0}^{+\infty} d \zeta\left(1+\frac{\zeta^{\{\Phi / 2 \pi\}} e^{-\zeta}}{\Gamma(\{\Phi / 2 \pi\})}-\frac{\Gamma(\{\Phi / 2 \pi\}, \zeta)}{\Gamma(\{\Phi / 2 \pi\})}\right)= \\
& =-\frac{1}{2}\left((1-\{\Phi / 2 \pi\})+\int_{0}^{+\infty} d \zeta\right),
\end{aligned}
$$

where $\Gamma(\alpha, \zeta)$ is the Upper Incomplete Gamma Function [36], with $\zeta=e B r^{2} / 2$. As a consequence each one of the fermion numbers picks up a non-vanishing vacuum expectation, namely $N_{+}=\left\langle 0\left|\hat{N}_{+}\right| 0\right\rangle=-\left(\frac{\Phi_{B}}{4 \pi}+\left\{\frac{\Phi}{4 \pi}\right\}\right)$ and $N_{-}=\left\langle 0\left|\hat{N}_{-}\right| 0\right\rangle=-\left(\frac{\Phi_{B}}{4 \pi}+\frac{1}{2}-\left\{\frac{\Phi}{4 \pi}\right\}\right)$, where $\Phi_{B} / 2 \pi$ is the reduced flux of the external magnetic field which is not quantized; it is 
infinity in fact once $B_{V}$ is uniform and in the calculations we have done so far the graphene sample has infinity dimensions $(r \in[0, \infty))$. As one can see, there is an induced fermion charge associated to each one of the valley due to the magnetic fields, whose results are compatible with the results of the induced electric charge and parity anomaly in QED in $2+1$ dimensions. In fact, the total induced charge of the system, which is also a physical observable and may be fractional when specific configurations of the magnetic and pseudomagnetic fields are considered and is fractional under Kekulé deformations - is

$$
Q=\langle 0|\hat{Q}| 0\rangle=-\frac{e}{2}\left(-1+2 e \frac{\Phi_{B}}{2 \pi}\right)
$$

From the above expression one sees that this induced charge is infinity once the flux of the external and uniform magnetic field is infinity, but it also has a fractional contribution $e / 2$ due to the A-B-like pseudomagnetic field.

On the other hand the induced valley number is finite and given by

$$
N_{\mathrm{v}}=\left\langle 0\left|\hat{N}_{\mathrm{v}}\right| 0\right\rangle=\left\langle 0\left|\hat{N}_{+}-\hat{N}_{-}\right| 0\right\rangle=\frac{1}{2}-\left\{\frac{\Phi}{2 \pi}\right\}
$$

That is a measure of the imbalance on the number of zero-modes among the valleys. Parenthetically we notice that we would have $N_{\mathrm{v}}=-1 / 2(1-\{\Phi / 2 \pi\})$ had we considered the zero-energy particles in the valence band.

Another consequence of the $c$-number as it stands in (18) and in (19) is that each particle in the conduction or in the valence band would carry a fractional valley charge as it follows

$$
\begin{gathered}
\hat{N}_{\mathrm{v}}\left|1_{\text {cond }}, n, m, \pm\right\rangle=\hat{N}_{\mathrm{v}} \mathbf{c}_{n, m, \pm}^{\dagger}|0\rangle=( \pm 1+1 / 2(1-\{\Phi / 2 \pi\}))\left|1_{\text {cond }}, n, m, \pm\right\rangle \\
\hat{N}_{\mathrm{v}}\left|1_{\mathrm{val}}, n, m, \pm\right\rangle=\hat{N}_{\mathrm{v}} \mathbf{d}_{n, m, \pm}^{\dagger}|0\rangle=(\mp 1+1 / 2(1-\{\Phi / 2 \pi\}))\left|1_{\mathrm{val}}, n, m, \pm\right\rangle
\end{gathered}
$$

From this last result one can see that, except for the zero-energy states, to each particle in the valence band and in the valley $+(-)$ is assigned the same valley charge of a particle in the conduction band and in the valley $-(+)$.

\section{FRACTIONAL (EVEN IRRATIONAL) SPIN POLARIZATION}

In this section we discuss the analogy between induced valley number and induced spin polarization in graphene in order to reinforce the development of valleytronics in analogy 
to spintronics. In valleytronics the pseudomagnetic fields due to topological defects, microstresses and deformations in clean graphene samples had shown to be useful in the filtering mechanism which selects valley-polarized electric currents as much as spin-polarized currents are produced in the context of spintronics.

In the previous two sections we have not considered the spin degrees of freedom, since in clean samples of graphene there is no room to spin-spin interaction or spin flipping in graphene. In the present section we decouple the two valley degrees of freedom from the full Dirac Hamiltonian that describes the low energy dynamics of electrons in the honeycomb lattice and we are left with the pseudospin (sublattices $a$ and $b$ ) and spin degrees of freedom. By following [29] we write the following quantum mechanics Dirac Hamiltonian operator

$$
\hat{h}=i(\vec{\sigma} \otimes I) \cdot\left[\vec{\nabla}-i e \vec{V}-i\left(I \otimes \tau_{3}\right) \vec{A}\right] .
$$

The $4 \times 4$ matrix structure of $\hat{h}$ comes from the direct product of $2 \times 2$ matrices, one of the sets, namely the identity matrix $I$ and the Pauli matrices $\sigma^{i}$ are associated to the pseudospin, while the other one is formed by the identity matrix and the spin Pauli matrices $\tau^{a}$. Moreover, $\vec{V}$ is the vector gauge potential associated to the external magnetic field and $\vec{A}$ is the axial-vector gauge potential associated to the pseudomagnetic field. In order to carry the analogy of induced valley number and induced spin polarization further and show that the spin polarization can be fractional as a consequence of the imbalance on the number of zero modes with different spin polarizations, we consider that the gauge potentials have the same configurations as given in (5).

Because $\hat{h}$ commutes with the spin polarization operator $I \otimes \tau_{3}$, one can label the energy eigenstates as $\Psi_{ \pm}=\frac{1}{2}\left(1 \pm \sigma_{3}\right) \otimes I \Psi$, where $\Psi_{+}$and $\Psi_{-}$are eigenstates of $I \otimes \tau_{3}$ also, with eigenvalues +1 and -1 , respectively. Moreover, $\hat{h}$ anticommutes with $\mathcal{C}=\sigma_{3} \otimes \tau_{3}$, then if $\Psi_{ \pm}^{E}$ is an eigenstate of $\hat{h}$ with eigenvalue $E \neq 0, \mathcal{C} \Psi_{ \pm}^{E}$ is also an eigenstate of $\hat{h}$ with eigenvalue $-E$, and the zero modes are $\mathcal{C}$ self-conjugate. With such considerations one can find the explicit form of the eigenstates of $\hat{h}, I \otimes \tau_{3}$ and $\hat{L}=-i \partial_{\theta}$ as they were found in section II. Moreover, according to the steps developed in the previous section, only the zero modes matters to the calculation of the vacuum expectation value. Then, for the sake of simplicity 
we present only them here, namely

$$
\begin{gathered}
\Psi_{0,+, l}=\sqrt{\frac{(e B / 2)^{1+l-\frac{\Phi}{2 \pi}}}{\pi \Gamma\left(1+l-\frac{\Phi}{2 \pi}\right)}}\left(\begin{array}{c}
e^{i l \theta} \\
0 \\
0 \\
0
\end{array}\right) r^{l-\frac{q \Phi}{2 \pi}} e^{-e B r^{2} / 2} \text { and } \\
\Psi_{0, k,-}(\vec{r})=\sqrt{\frac{(e B / 2)^{1+k+\frac{\Phi}{2 \pi}}}{\pi \Gamma\left(1+k+\frac{\Phi}{2 \pi}\right)}}\left(\begin{array}{c}
0 \\
e^{i k \theta} \\
0 \\
0
\end{array}\right) r^{k+\frac{\Phi}{2 \pi}} e^{-\frac{e B}{4} r^{2}}
\end{gathered}
$$

We notice that the only thing that distinguishes these zero-energy eigenstates from those in (66) is their matrix structure. The same happens to the other eigenstates. In other words, eigenstates $\Psi_{|E|,+, l}\left(\Psi_{|E|,-, k}\right)$ have in general the first and the third (the second and the fourth) components different from zero. The energy eigenvalues are given by (11) and (12).

Since the quantum field operator

$$
\hat{S}=\frac{1}{2} \int d^{2} \vec{r}\left[\hat{\Psi}^{\dagger}(\vec{r}, t), I \otimes \tau_{3} \hat{\Psi}^{\dagger}(\vec{r}, t)\right]
$$

commutes with the quantum field theory Hamiltonian operator

$$
\hat{H}=\frac{1}{2} \int d^{2} \vec{r}\left[\hat{\Psi}^{\dagger}(\vec{r}, t), i(\vec{\sigma} \otimes I) \cdot\left[\vec{\nabla}-i e \vec{V}-i\left(I \otimes \tau_{3}\right) \vec{A}\right] \hat{\Psi}^{\dagger}(\vec{r}, t)\right]
$$

it can be associated to a physical observable. Then, by following the same steps of the section III one finds the induced spin polarization as given by

$$
S=\langle 0|\hat{S}| 0\rangle=\frac{1}{2}-\left\{\frac{\Phi}{2 \pi}\right\} .
$$

One can check that the (quasi)particles would carry fractional (even irrational) spin polarization. This result must be understood only as an illustrative analogy to the result on the fractional valley number due to topological defects in the graphene lattice, because topological defects do not affect the spin polarization degree of freedom.

\section{FURTHER COMMENTS AND CONCLUSIONS}

We have analyzed the influence of an external magnetic field in a clean graphene sheet with a local topological defect (single disclination). Such a topological defect can be described in the low-energy continuum model for the charge carriers as a Aharonov-Bohm-like 
pseudomagnetic field whose axial-vector gauge potential couples to the two valley degrees of freedom with different signs. As a consequence of this and due to the configuration of the pseudomagnetic field one has a partial breaking of the valley symmetry originally present in the low-energy effective Hamiltonian for the charge carriers. This partial breaking of the valley symmetry is revealed in the energy spectrum of the system which is no longer the usual (degenerate) Landau levels (LL) for a (relativistic) massless fermion in a uniform magnetic field. For instance, between the zero-energy level and the first LL with energy $\sqrt{2 e B}$, there appears two additional non-degenerate energy levels with energies $\sqrt{2 e B\{\Phi / 2 \pi\}}$ and $\sqrt{2 e B(1-\{\Phi / 2 \pi\})}(0<\{\Phi / 2 \pi\}<1$ is the fractional part of the reduced pseudomagnetic flux), each one of these intermediary energy levels contains a unique member of only one of the valleys. In addition, each one of the LL is partially degenerate as regards the valley degree of freedom, namely there is an imbalance on the number of valley states on those energy levels. A measure of this imbalance, which we have called a partial filtering, can be defined in the context of quantum field theory as the valley number given by the vacuum expectation value of the number operator associated to one of the valleys minus the expectation value of the number operator associated to the other valley. We have analyzed that in the third section and have shown that due to the sole contribution of the zero modes, the valley number is in fact finite and may be fractional (even irrational) for $\{\Phi / 2 \pi\} \neq 1 / 2$. To show that the sum of states is infinity, specifically concerning the zero-energy states, we have also computed the induced electric charge and have shown that it is proportional do the flux of the external magnetic field, which is infinity because the external field is uniform and the graphene sample is taken to be infinity. This last result would be expected from the parity anomaly in QED in $2+1$ space-time dimensions. In fact, in the absence of intervalley scattering, the continuum model for the dynamics of charge carriers in graphene in magnetic and pseudomagnetic fields may be seen as two decoupled QED in $2+1$ dimensions (one for each valley degree of freedom) for massless fermions.

The above splitting on the lowest LL may be a good candidate to explain the degeneracy lift of the zero-energy level and, perhaps the appearance of a mass gap, that has been observed in graphene samples under relatively strong magnetic fields. Since it seems to be very difficult to have a perfect, free-of-defects graphene sample, the influence of those kind of topological defects enhanced by a relatively strong magnetic field might be observed.

We have also wondered if similar effects could also take place in strained samples of 
graphene under microstresses, as those reported in [10] where uniform (pseudo)magnetic fields up to $300 T$ seems to be realized. In view of the results in [25], we conclude that particles associated to the different valleys would describe orbits with different cyclotron frequencies, namely $\omega_{ \pm}=\left|e B \pm B_{A}\right| / 2$ and each Landau level, say $\sqrt{2 e B n}$, is split in two levels with energies $\sqrt{\left|e B+B_{A}\right| n}$ and $\sqrt{\left|e B-B_{A}\right| n}$, each one contains representative of only one of the valleys. The zero-energy level still persists with representatives of both valleys, but since they have different cyclotron frequencies, there will be an induced valley number given by $N_{\mathrm{v}}= \pm \Phi / 2 \pi$ (the ambiguity of the sign is attributed to the zero-energy particles be assigned to the valence or to the conduction band), which is no longer fractional, neither is finite once the pseudomagnetic field is uniform and the sample is taken to be infinitely large. An interesting aspect on these results is that now we are able, by fine-tuning the external magnetic field, to filter one of the valleys completely and leading to valley-polarized cyclotron orbits and to chiral eddy currents.

We have also analyzed other kinds of deformations in graphene, such as the symmetric in-plane ones [18], but we have not been able to reach a definite conclusion in favor of partial filtering in this case. Nevertheless, in view of the results presented here and based on the index theorems on the number of zero modes of the Dirac Hamiltonian [37]-[40], we believe that a partial valley-filtering and a non-null induced valley number take place whenever there is a net pseudomagnetic flux. Then, for the example of in-plane deformation analyzed

in [18], where the pseudomagnetic field is given by $B_{A}=\frac{-\kappa u_{00} \Phi_{0}}{\pi \sigma^{6}}\left(r^{4}-7 \sigma^{2} r^{2}+4 \sigma^{4}\right) e^{-r^{2} / 2 \sigma^{2}}$ we have found the pseudomagnetic flux as $\Phi=4 \kappa u_{00} \Phi_{0}$, and we would expect that, even in the background of the uniform magnetic field for which the index theorem seems not to be applicable since one has an infinite flux [41], an induced valley number given by $N_{\mathrm{v}}=$ $\pm 2 \kappa u_{00} \Phi_{0}$, where $\Phi_{0}$ is the flux quantum and $\kappa u_{00}=0.3$ from the data in [18].

\section{ACKNOWLEDGMENTS}

AEO thanks to the CAPES/CNPq-IEL Nacional-Brasil program for the scholarship. This work is also partially supported by CNPq (procs. 482043/2011-3, 306316/2012-9). 
[1] C.-Y. Hou, C. Chamon, and C. Mudry, Phys. Rev. Lett. 98, 186809 (2007).

[2] P. R. Wallace, Phys. Rev. 71, 622 (1947).

[3] G. W. Semenoff, Phys. Rev. Lett. 53, 2449 (1984).

[4] R. Jackiw and S.-Y. Pi, Phys. Rev. Lett. 98, 266402 (2007).

[5] J. Gonzalez, F. Guinea, and M. A. H. Vozmediano, Phys. Rev. Lett. 69, 172 (1992); J. Gonzalez, F. Guinea, and M. A. H. Vozmediano, Nucl. Phys. B 406, 771 (1993).

[6] J. K. Pachos, M. Stone, and K. Temme, Phys. Rev. Lett. 100, 156806 (2008).

[7] C. Chamon, C.-Y. Hou, R. Jackiw, C. Mudry, S.-Y. Pi, and G. Semenoff, Phys. Rev. B 77, 235431 (2008).

[8] I. F. Herbut, Phys. Rev. B 81, 205429 (2010).

[9] S. Deser, R. Jackiw, and S. Templeton, Phys. Rev. Lett 48, 975 (1982); and Ann. Phys. (N.Y.) 140, $372(1982)$.

[10] N. Levy, S. A. Burke, K. L. Meaker, M. Panlasigui, A. Zettl, F. Guinea, A. H. Castro Neto, and M. F. Crommie, Science 329, 544 (2010).

[11] J. C. Meyer, A. K. Geim, M. I. Katsnelson, K. S. Novoselov, T. J. Booth, and S. Roth, Nature 446, 60 (2007).

[12] A. Cortijo and M. A. H. Vozmediano, Nucl. Phys. B 763, 293 (2007).

[13] F. deJuan, A. Cortijo, and M. A. H. Vozmediano, Phys. Rev. B 76, 165409 (2007).

[14] Y. A. Sitenko and N. D. Vlassi, Nucl. Phys. B 787, 241 (2007).

[15] F. Guinea, M. I. Katsnelson, and M. A. H. Vozmediano, Phys. Rev. B 77, 075422 (2008).

[16] F. Guinea, B. Horovitz, and P. LeDoussal, Phys. Rev. B 77, 205421 (2008).

[17] E.-A. Kim and A. H. Castro Neto, Eur. Phys. Lett. 84, 57007 (2008).

[18] F. De Juan, A. Cortijo, M. A. H. Vozmediano, and A. Cano, Nat. Phys. 7, 810 (2011).

[19] M. A. H. Vozmediano, M. I. Katsnelson, and F. Guinea, Phys. Rep. 496, 109 (2010).

[20] A. Cortijo, Doctorate Thesis in Physics Sciences, Universidad Carlos III de Madrid, 2007.

[21] T. Fujita, M. B. A. Jalil, S. G. Tan, and S. Murakami, J. Appl. Phys. 110, 121301 (2011).

[22] F. Zhai, X. Zhao, K. Chang, and H. Q. Xu, Phys. Rev. B 82, 115442 (2010).

[23] Fujita, M. B. A. Jalil, and S. G. Tan, Appl. Phys. Lett. 97, 043508 (2010).

[24] A. Rycerz, J. J. Tworzydlo, and C. W. J. Beenakker, Nat. Phys. 3, 172 (2007). 
[25] A. E. Obispo and M. B. Hott, Phys. Rev. B 89, 165405 (2014).

[26] F. D. M. Haldane, Phys. Rev. Lett 61, 2015 (1988).

[27] S. Azevedo, C. Furtado, and F. Moraes, Phys. Stat. Sol. B 207, 387 (1998).

[28] A. Cortijo and M. A. H. Vozmediano, Nucl. Phys. B 763, 293 (2007).

[29] G.W. Semenoff, Phys. Rev. B 83, 115450 (2011).

[30] C. R. Hagen, Phys. Rev. Lett. 64, 503 (1990).

[31] C. R. Hagen, Phys. Rev. D. 48, 5935 (1993).

[32] U. Percoco and V. M. Villalba, Phys. Lett. A 140, 105 (1989).

[33] Yu. A. Sitenko, Phys. Rev. 60, 125017 (1999). Yu. A. Sitenko, Ann. Phys. (N.Y.) 282, 167 (2000).

[34] V. G. Bagrov. D. M. Gitman, and V. B. Tlyachev, J. Math. Phys. 42, 1933 (2001).

[35] S. P. Gavrilov, D. M. Gitman, and A. A. Smirnov, Eur. Phys. J. C 32, 119 (2003).

[36] M. Abramowitz and I. A. Stegun, Handbook of Mathematical Functions (Dover, New York, 1965).

[37] M. Atiyah and I. Singer, Ann. Math 87, 484 (1964). For a review, see T. Eguchi, R. Gilkey, and A. Hanson, Phys. Rep. 66, 213 (1980). See also

[38] Y. Aharonov and A. Casher, Phys. Rev. A 19, 2461 (1979).

[39] E. J. Weinberg, Phys. Rev. D 24, 2669 (1981).

[40] R. Jackiw, Phys. Rev. D 29, 2375 (1984).

[41] A. J. Niemi and G. W. Semenoff, Phys. Rep. 135, 99 (1986). 